\title{
3D-Printed Graphene Antennas and Interconnections for Textile RFID Tags: Fabrication and Reliability towards Humidity
}

\author{
Han He, Mitra Akbari, Lauri Sydänheimo, Leena Ukkonen, and Johanna Virkki
}

BioMediTech Institute and Faculty of Biomedical Sciences and Engineering, Tampere University of Technology, P.O. Box 692, 33101 Tampere, Finland

Correspondence should be addressed to Han He; han.he@tut.fi

Received 7 April 2017; Accepted 15 May 2017; Published 5 June 2017

Academic Editor: Sanming Hu

Copyright (C) 2017 Han He et al. This is an open access article distributed under the Creative Commons Attribution License, which permits unrestricted use, distribution, and reproduction in any medium, provided the original work is properly cited.

\begin{abstract}
We present the possibilities of 3D direct-write dispensing in the fabrication of passive UHF RFID graphene tags on a textile substrate. In our method, the graphene tag antenna is deposited directly on top of the IC strap, in order to simplify the manufacturing process by removing one step, that is, the IC attachment with conductive glue. Our wireless measurement results confirm that graphene RFID tags with printed antenna-IC interconnections achieve peak read ranges of 5.2 meters, which makes them comparable to graphene tags with epoxy-glued ICs. After keeping the tags in high humidity, the read ranges of the tags with epoxy-glued and printed antenna-IC interconnections decrease 0.8 meters and 0.5 meters, respectively. However, after drying, the performance of both types of tags returns back to normal.
\end{abstract}

\section{Introduction}

Graphene is an environmentally friendly and low-cost conductive material that has the capacity to integrate with challenging substrate materials, such as soft and stretchable textiles $[1,2]$. For those reasons, and because of graphene's great electrical and mechanical properties, graphene-based conductive inks have the potential to revolutionize the area of printed electronics, by replacing traditional inks with metallic components.

3D direct-write dispensing is a fast and versatile additive manufacturing method, which enables the printing of complex geometries into textile materials with micron resolution accuracy [3]. Graphene ink can be used as a conductor in these printed structures, which opens novel possibilities for textile antenna and antenna-electronics interconnection fabrication. In particular, passive RFID (radio-frequency identification) tags integrated into textiles, which will be the focus of this paper, provide versatile wireless identification and sensing possibilities embedded into clothing [4-7] and are thus a great platform for this new fabrication method and material. In wearable systems, textile antennas can be used in a great variation of applications [8] and often they operate in an extremely challenging environment. Wearable applications require the antennas and interconnections to endure different environmental stresses, such as moisture and wetting $[9,10]$.

In this paper, the possibilities of 3D-printed graphene antennas and antenna-IC (integrated circuit) interconnections are studied, in order to simplify the manufacturing process of passive UHF (ultra high frequency) RFID tags on a traditional $100 \%$ cotton fabric. By printing the graphene antennas directly on top of IC fixture, there is no need for a separate IC attachment step, which means more cost- and time-effective fabrication of these wireless components. The wireless performance of these tags is evaluated in normal room conditions, as well as in high humidity conditions, and compared to tags were the IC is attached with conductive glue.

\section{Manufacturing of Passive UHF RFID Tags}

In this work, 3D-printing technique is used to fabricate graphene-based RFID tags on a 100\% cotton fabric by using a water-based graphene ink (HDPlas ${ }^{\circledR}$ IGSC02002) [11]. The printing is done with nScrypt tabletop series $3 \mathrm{D}$ direct-write dispensing system, and the main manufacturing parameters are defined in Table 1. The printing spacing and angle can 


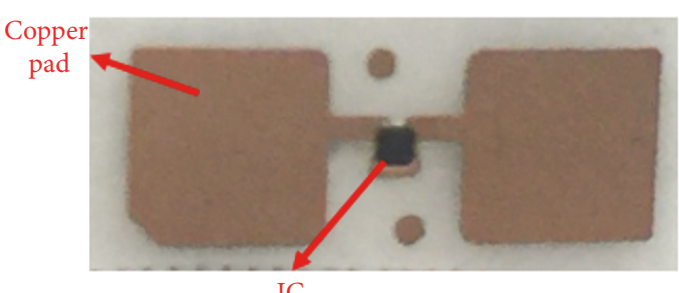

IC

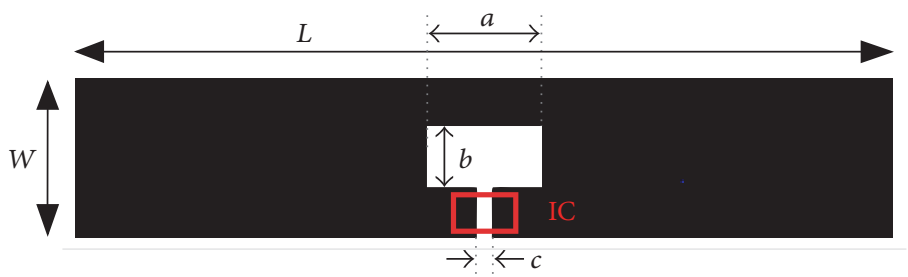

(a)

(b)

\begin{tabular}{ccccc}
\hline$a(\mathrm{~mm})$ & $b(\mathrm{~mm})$ & $c(\mathrm{~mm})$ & $L(\mathrm{~mm})$ & $W(\mathrm{~mm})$ \\
\hline 14.3 & 8.125 & 2 & 100 & 20 \\
\hline
\end{tabular}

Figure 1: The IC strap (a) and the tag antenna structure (b).

TABLE 1: The used 3D-printing and curing parameters.

\begin{tabular}{lc}
\hline Parameter & \\
\hline Material feed pressure & 16.9 Psi \\
Printing spacing & 125 microns \\
Printing angle & $0^{\circ}$ \\
Inner diameter of tip & 125 microns \\
Number of printed layers & 1 \\
Curing & $60^{\circ} \mathrm{C}, 30$ minutes \\
\hline
\end{tabular}

be defined when designing the printed pattern by a built-in software. The air pressure from a positive pressure pump is applied to the system, which pushes the ink into the main valve body and finally through the ceramic nozzle tip. By adjusting a constant material pressure, running a customizable computer-controlled valve open and close process, and selecting the ceramic nozzle tip with suitable size, the printing system can produce a controllable ink flow, precise starts and stops, and the ability to utilize a wide range of material viscosities [12].

Two different methods are studied for the antenna-IC interconnection. The first method is a previously reported way, where the IC is attached on top of the printed and cured antenna with conductive silver epoxy (Circuit Works CW2400). This method has been used, for example, in $[2,13]$. In the second method, the antenna is deposited on top of the IC fixture, and thus the antenna-IC interconnection is cured together with the antenna. This 3D-printing approach skips one process step and thus saves significant amounts of time and costs. In both cases, only one layer of ink is printed. The curing of the antennas is done in $60^{\circ} \mathrm{C}$ for 30 minutes, as guided by the ink datasheet [11].

The utilized IC strap (NXP UCODE G2iL series RFID IC, provided by the manufacturer in a strap) and the tag antenna structure are shown in Figure 1. The antenna is quite wide $(2 \mathrm{~cm})$, which reduces the impact of imperfections in the print outcome, and the length of the antenna $(10 \mathrm{~cm})$ is sufficient to avoid the weaknesses of electrically small antennas in the UHF frequencies from $850 \mathrm{MHz}$ to $1000 \mathrm{MHz}$. This antenna geometry has been previously used successfully on textile tags $[2,3]$. The simulated current distribution on the dipole antenna at $915 \mathrm{MHz}$ is shown in Figure 2.

For each tag type, three samples are fabricated to also evaluate the reproducibility. The surface of a 3D-printed and cured graphene antenna is shown in Figure 3. As can be seen, the graphene ink layer has a good adherence to the fabric substrate. Ready tags with epoxy-glued and 3D-printed antenna-IC interconnections on a cotton fabric are shown in Figures 4(a) and 4(b), respectively.

\section{Measurements}

The wireless performance of the fabricated tags is evaluated with a Tagformance RFID measurement system. All the measurements are conducted with the tag suspended on a foam fixture in an anechoic chamber. The measurements were obtained from a fixed angle (the angle of the highest read range) between the reader antenna and tag. In all measurements, the longer side of the antenna, where the IC was attached, was directly facing the reader antenna. During the test, we record the lowest continuous-wave transmission power (threshold power) at which the tag remains responsive. The forward loss from the transmit port to the tag is first attained using a reference tag with known properties. The measurement equipment calculates the theoretical read range of the tag using its measured threshold power along with the measured forward losses. The theoretical read range is based on the relation given in

$$
d_{\mathrm{Tag}}=\frac{\lambda}{4 \pi} \sqrt{\frac{\mathrm{EIRP}}{P_{\mathrm{TS}} L_{\mathrm{fwd}}}}
$$

where $\lambda$ is the wavelength transmitted from the reader antenna, $P_{\mathrm{TS}}$ and $L_{\mathrm{fwd}}$ are the measured threshold power and forward losses, correspondingly, and EIRP is the emission limit of an RFID reader given as equivalent isotropic radiated power. All the results correspond to EIRP $=3.28 \mathrm{~W}$, which is the emission limit in European countries.

Finally, the effects of moisture on the tags' wireless performance are investigated. During the moisture test, the tags (whole tag, including the IC) are placed in tap water and wirelessly measured before the test and immediately after 1 


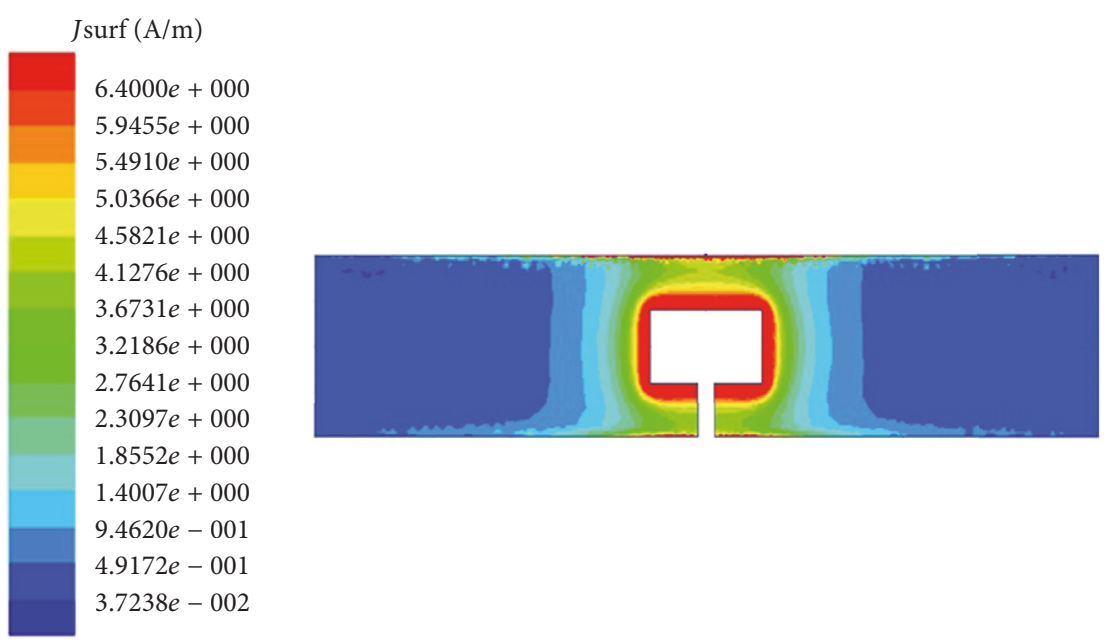

Figure 2: The simulated current distribution on the antenna at $915 \mathrm{MHz}$.

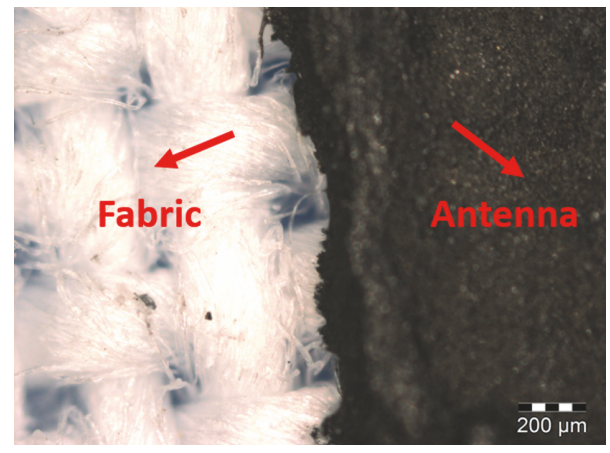

FIGURE 3: Optical microscopic image of a 3D-printed graphene antenna on cotton (magnification $\times 20$ ).

minute in water. The tags are then left in room conditions to dry and are measured again after 1 hour, 1 day, and finally 1 week, when they are totally dry.

\section{Achieved Results}

On porous substrates, such as fabric, the ink is partially absorbed into the substrate and the average thickness of the printed layer depends thus on the substrate material. Figure 5 shows a microscopic image of a cross-section of a 3D-printed tag antenna together with the measured thickness. The average thickness of all fabricated antenna samples is $610.15 \mu \mathrm{m}$, while the standard deviation is $23.51 \mu \mathrm{m}$. Moreover, the average sheet resistances of all the fabricated antenna samples in room conditions and after 1 minute in water are $6.47 \Omega / s q$ and $9.51 \Omega /$ sq, respectively, while the corresponding standard deviations are $1.45 \Omega /$ sq and $2.27 \Omega /$ sq.

Figure 6 presents the attainable read ranges of the 3Dprinted tags before and after the high humidity conditions. Despite the variation in the antenna thickness, all similarly fabricated tags showed similar performance. As can be seen, the peak read ranges of the tags with printed antenna-IC joints can achieve 5.2 meters, which is about 0.4 meters higher than the read ranges of the tags with epoxy-glued joints. Thus, the 3D-printed electric interconnection can be considered to be a good replacement for the epoxy-glued one. However, in order to draw further conclusions, the effects of antenna thickness variation on the tag read range should be studied with a significantly larger amount of antenna samples. Also, the contact resistances of the printed and glued IC attachments need to be investigated, and especially the variation of hand-made glue connections can affect the read ranges.

Both types of tags also show better performance than earlier published results using the same antenna geometry and conductive ink [2], where the graphene tag antennas were fabricated by doctor-blading on a cotton fabric substrate and the IC was attached with conductive epoxy. The same antenna geometry has been previously also reported to be 3D-printed using silver and copper inks [3]. The achieved peak read ranges for the copper- and silver-based tags were around 6 and 11 meters, respectively. Thus, the graphene ink cannot yet offer comparable read ranges to silver-based inks, but the achieved read ranges are suitable for many RFID identification and sensing applications. Also, it is interesting to study the effect of antenna thickness, for example, antennas with two or more layers of ink, on the tag performance.

Based on the moisture test, the read ranges of both types of tags decrease after 1 minute in high humidity conditions, which is also supported by the fact that the sheet resistances of the graphene antennas increase as the humidity level increases. After keeping the tags in high humidity, the read ranges of the tags with the epoxy-glued and printed antennaIC interconnections decrease 0.8 meters and 0.5 meters, respectively. The effect of humidity on the tags with the epoxy-glued ICs is more significant, most probably due to the moisture absorption of the glue itself, which affects the antenna-IC impedance matching. This finding strongly supports the use of the printed antenna-IC joint. For the tags with the printed IC attachment, the read range in the frequency range of $850-900 \mathrm{MHz}$, after the humidity test, is better than that in the dry state. It could be true that despite 


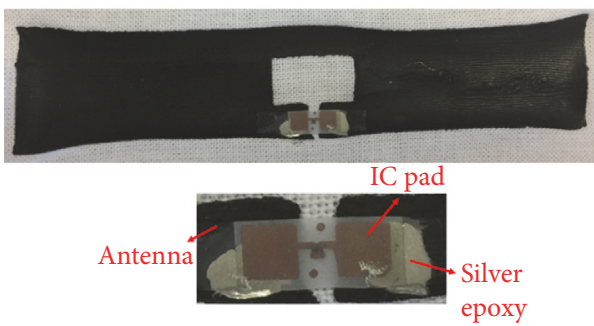

(a)

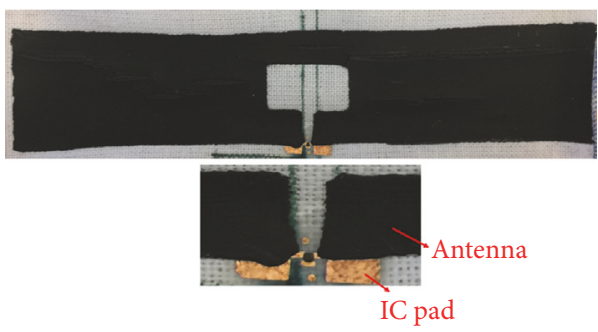

(b)

FIGURE 4: Ready 3D-printed passive UHF RFID tags and magnifications of the antenna-IC interconnections of (a) epoxy-glued joint and (b) printed joint.

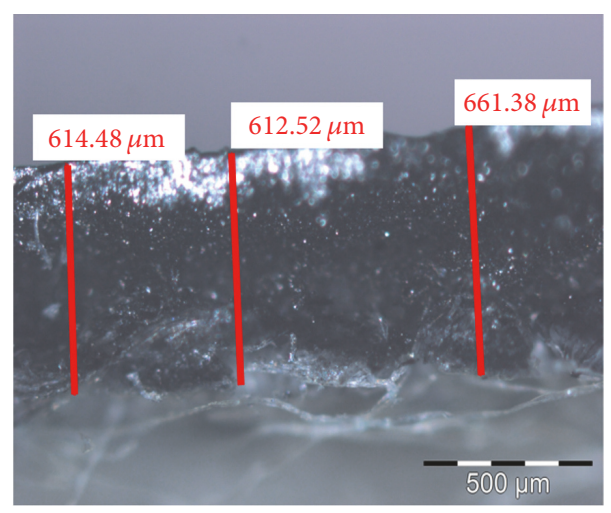

FIGURE 5: A cross-section of a printed antenna and its thickness.

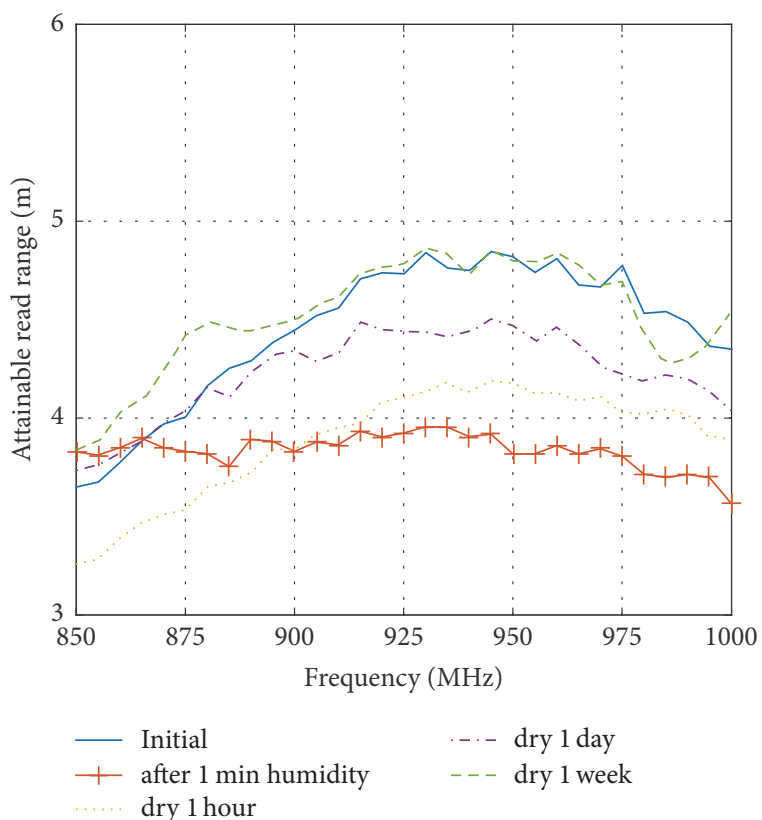

(a)

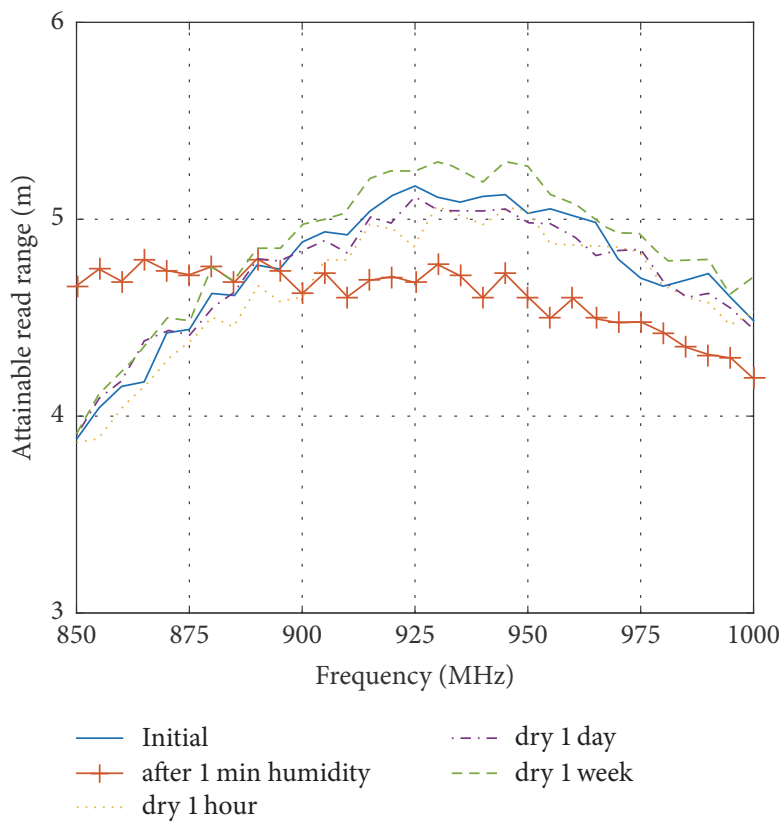

(b)

FIGURE 6: Read ranges of graphene RFID tags before and after high humidity conditions: (a) epoxy-glued IC attachment and (b) printed IC attachment. 
the increased ohmic losses caused by the water, the read range in that frequency range increases due to the changed antenna impedance and power reflection from the antennaIC interface. It should be noted that humidity can also have an effect on the fabric substrate. The moisture can affect the dielectric constant and loss tangent of the fabric, which can affect the wireless performance of the tags.

After drying for one hour in room conditions, the read ranges of both types of tags gradually increase back to normal. After 1 week, when the tags are totally dry, the performance of both types of tags has returned close to the initial performance. Thus, the harsh moisture test did not have a permanent effect on the performance of these tags.

\section{Conclusions}

In this paper, the possibility of applying 3D direct-write dispensing to form the antenna-IC connection together with the antenna was studied in order to simplify the manufacturing process of passive graphene UHF RFID tags on cotton fabric. The results were compared to tags fabricated with epoxy-glued ICs. Also, the effects of moisture on the tag performance were investigated. Based on our results, the tags with the 3D-printed antenna-IC interconnections showed excellent wireless performance and read ranges comparable to the tags with the epoxy-glued ICs. Moreover, moisture did not have a significant detrimental effect on either type of tags, and the slightly decreased performance returned back to normal when the tags were dry again. These findings support the use of 3D-printing in fabrication of graphene antennas and the use of the printed antenna-IC attachment method. However, more work needs to be done in order to draw more extensive conclusions.

\section{Conflicts of Interest}

The authors declare that there are no conflicts of interest regarding the publication of this paper and that the mentioned received funding in Acknowledgments did not lead to any conflicts of interest regarding the publication of this manuscript.

\section{Acknowledgments}

This research work was supported by the Jane and Aatos Erkko Foundation, Academy of Finland, and TEKES.

\section{References}

[1] X. Huang, T. Leng, X. Zhang et al., "Binder-free highly conductive graphene laminate for low cost printed radio frequency applications," Applied Physics Letters, vol. 106, no. 20, Article ID 203105, 2015.

[2] M. Akbari, J. Virkki, L. Sydanheimo, and L. Ukkonen, "Toward graphene-based passive UHF RFID textile tags: A Reliability Study," IEEE Transactions on Device and Materials Reliability, vol. 16, no. 3, pp. 429-431, 2016.

[3] T. Björninen, J. Virkki, L. Sydänheimo, and L. Ukkonen, "Possibilities of 3D direct write dispensing for textile UHF
RFID tag manufacturing," in Proceedings of the IEEE International Symposium on Antennas and Propagation \& USNC/URSI National Radio Science Meeting, pp. 1316-1317, Vancouver, BC, Canada, July 2015.

[4] C. Occhiuzzi, G. Contri, and G. Marrocco, "Reading range of wearable textile RFID tags in real configurations," in Proceedings of the 5th European Conference on Antennas and Propagation (EUCAP '11), pp. 433-436, April 2011.

[5] R. Nayak, A. Singh, R. Padhye, and L. Wang, "RFID in textile and clothing manufacturing: technology and challenges," Fashion and Textiles, vol. 2, no. 1, 2015.

[6] D. Patron, W. Mongan, T. P. Kurzweg et al., "On the Use of Knitted Antennas and Inductively Coupled RFID Tags for Wearable Applications," IEEE Transactions on Biomedical Circuits and Systems, vol. 10, no. 6, pp. 1047-1057, 2016.

[7] L. Catarinucci, R. Colella, D. De Donno, and L. Tarricone, "Fully-passive devices for RFID smart sensing," in Proceedings of the IEEE Antennas and Propagation Society International Symposium (APSURSI '13), pp. 2311-2312, IEEE, Orlando, Fla, USA, July 2013.

[8] M. Stoppa and A. Chiolerio, "Wearable electronics and smart textiles: a critical review," Sensors, vol. 14, no. 7, pp. 11957-11992, 2014.

[9] C. Hertleer, A. Van Laere, H. Rogier, and L. Van Langenhove, "Influence of relative humidity on textile antenna performance," Textile Research Journal, vol. 80, no. 2, pp. 177-183, 2010.

[10] R. Salvado, C. Loss, R. Gonçalves, and P. Pinho, "Textile materials for the design of wearable antennas: a survey," Sensors, vol. 12, no. 11, pp. 15841-15857, 2012.

[11] S. Goodfellow, "Screen printable functionalized graphene ink," 2017, http://www.goodfellowusa.com/news-article/ screen-printable-functionalized-graphene-ink/.

[12] Nscrypt USA, “Smart Pump," 2017, http://www.nscrypt.com/ wp-content/uploads/2017/02/2016-SmartPump-Gen2.pdf.

[13] J. Virtanen, J. Virkki, L. Ukkonen, and L. Sydänheimo, "Inkjetprinted UHF RFID tags on renewable materials," Advances in Internet Things, vol. 2, no. 4, pp. 79-85, 2012. 


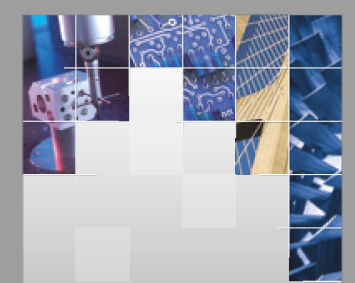

\section{Enfincering}
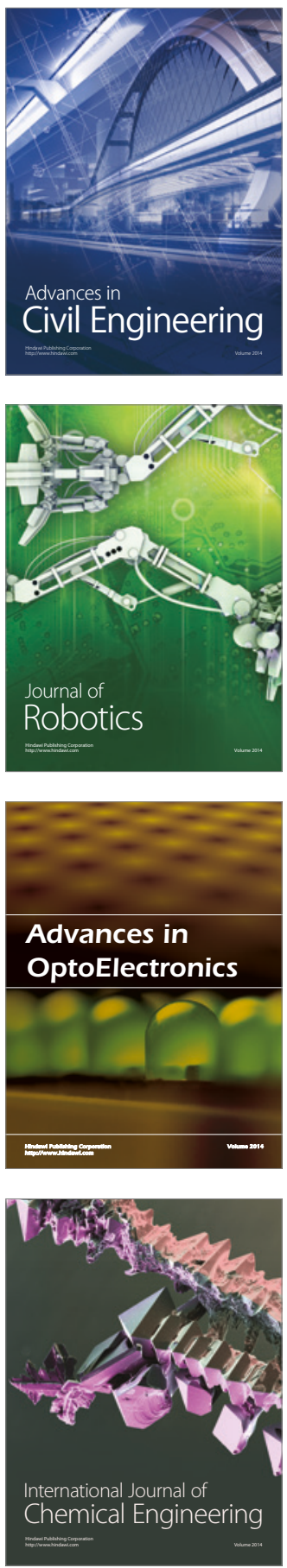

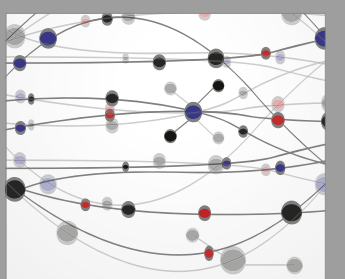

The Scientific World Journal

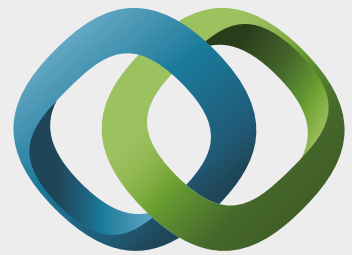

\section{Hindawi}

Submit your manuscripts at

https://www.hindawi.com
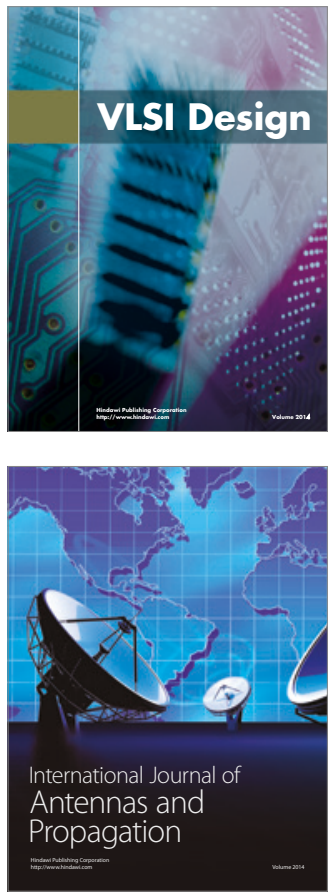

\section{Rotating}

Machinery
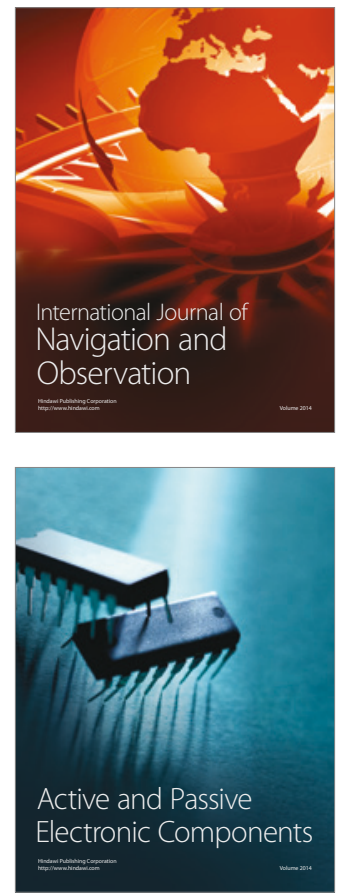
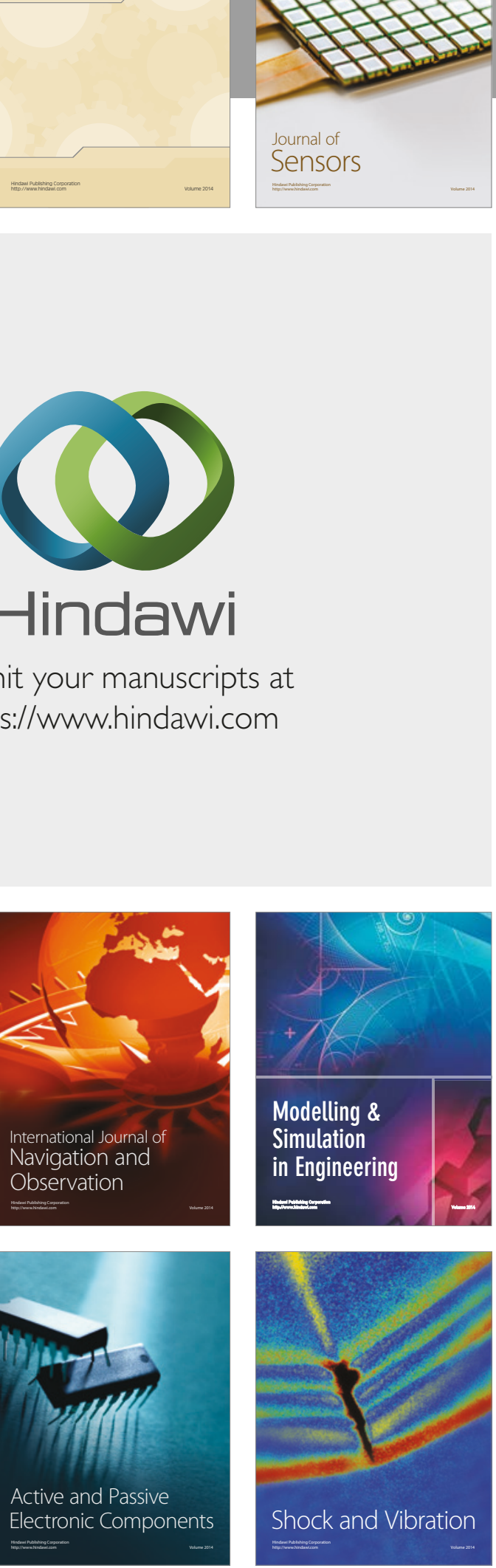
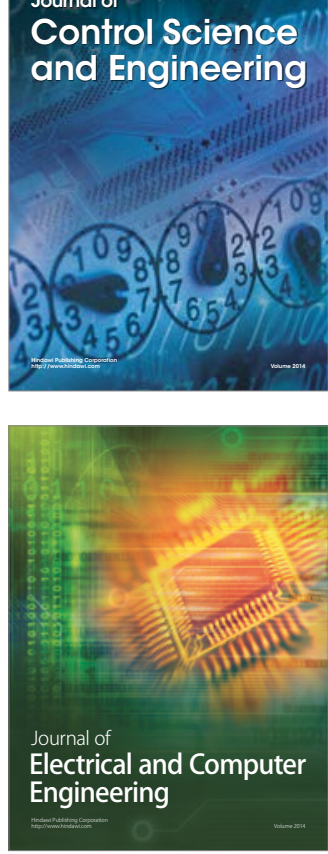

Distributed

Journal of

Control Science

and Engineering
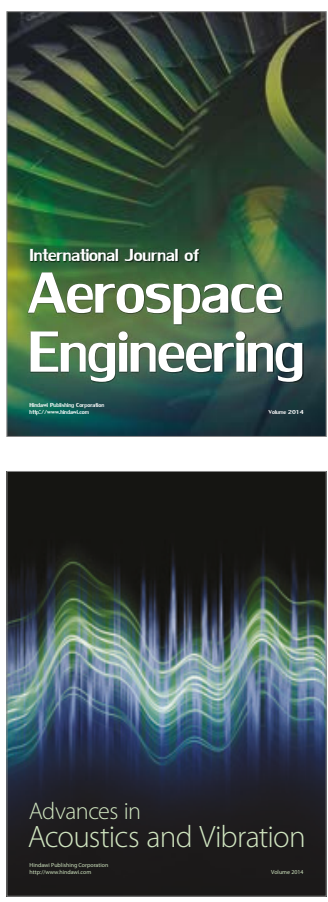

Sensor Networks 